Int. J. Dev. Biol. 54: 919-924 (2010)

doi: $10.1387 / \mathrm{ijdb} .092916 \mathrm{vm}$

\title{
Identification and gastrointestinal expression of Xenopus laevis FoxF2
}

\author{
VALÉRIE A. McLIN ${ }^{*}, 1,2$, RINA SHAH ${ }^{3}$, NEEKITA P. DESAI ${ }^{1}$ and MILAN JAMRICH ${ }^{3,4}$ \\ ${ }^{1}$ Department of Pediatrics, Section of Gastroenterology, Hepatology and Nutrition, Baylor College of Medicine, \\ ${ }^{2}$ Département de l'Enfant et de l'Adolescent, Unité de Gastroentérologie Pédiatrique, \\ Hôpitaux Universitaires de Genève, Switzerland, ${ }^{3}$ Department of Cellular and Molecular Biology and \\ ${ }^{4}$ Department of Molecular and Human Genetics, Baylor College of Medicine, Houston, TX, USA.
}

\begin{abstract}
FoxF genes are essential for visceral mesoderm development from Drosophila to human. However, part of the difficulty of studying the visceral mesoderm is its relative inaccessibility during early development. Owing to its external development Xenopus laevis presents considerable advantages for the study of visceral mesoderm formation, yet FoxF2 has not been identified in this system. Here, we describe the cloning and expression pattern of XFoxF2 during embryonic development, metamorphosis and adulthood, and compare and contrast it to the expression of FoxF1 in Xenopus laevis and FoxF2 in mouse.
\end{abstract}

KEY WORDS: Xenopus, FoxF2, visceral mesoderm, intestine, metamorphosis

The FoxF family of forkhead genes are a highly conserved group of genes necessary for visceral mesoderm development from Drosophilato humans (Clevidence et al., 1993, Mahlapuu et al., 2001, Mahlapuu et al., 1998, Zaffran et al., 2001). In Drosophila, mutant embryos null for the Foxfhomologue biniou, do not form visceral mesoderm (Zaffran et al., 2001). In mouse, Foxf1 null embryos die before embryonic day 10 from impaired extra-embryonic membrane and vascular development (Mahlapuu et al., 2001). However, Foxf1 heterozygous animals develop to term with a perinatal mortality of $90 \%$ owing to lung and foregut abnormalities (Mahlapuu et al., 2001). In mouse, there is a second Foxfgene: Foxf2. Like Foxf1, it is also expressed in the developing gastrointestinal tract, predominantly in the hindgut (Ormestad et al., 2004). However, Foxf2 expression is more diffuse, while Foxf1 expression is confined to epithelial-mesenchymal interfaces(Ormestad et al., 2004). Moreover, its expression is identified in the oral mesenchyme, presumptive genitalia, and developing limbs. Importantly, it is not expressed in the extraembryonic membranes, allowing for the study of its role during organogenesis since Foxf2/- mice develop to term (Ormestad et al., 2004). The two proteins are very similar in their DNA-binding domains and their C-termini, but otherwise divergent (Pierrou et al., 1994). Accordingly, evidence from murine experiments suggests that in spite of structural similarities and overlapping expression domains, they have distinct functions (Ormestad et al., 2006).

Xenopus laevis presents considerable advantages for the study of visceral mesoderm formation for several reasons. First, its development is external, which is advantageous for the study of early patterning events and tissue interactions. Second, Xenopus metamorphosis is characterized by extensive remodeling of the intestine associated with profound architectural changes, involving proliferation of the mesodermally-derived mesenchyme and muscularis. In Xenopus laevis, FoxF1 is expressed in the lateral plate mesoderm and the head mesenchyme (El-Hodiri et al., 2001). FoxF1 targeted knockdown using antisense morpholinos-oligonucleotides leads to severe defects in gut elongation and coiling at least in part due to abnormal cell proliferation of the lateral plate mesoderm (Tseng et al., 2004). Yet, FoxF2, which, based on mouse studies, would be hypothesized to also play an important role during gut development, has not been identified in this system. Here, we describe the cloning and expression pattern of FoxF2 during embryonic development, metamorphosis, and adulthood, and compare and contrast it to the expression of FoxF1 in Xenopus laevis and mouse.

Abbreviations used in this paper: vm, visceral mesoderm.

\footnotetext{
*Address correspondence to: Valérie McLin. Unité de Gatroentérologie Pédiatrique, Département de l'Enfant et de l'Adolescent, Hôpitaux Universitaires de Genève, 1211 Geneva, Switzerland. Fax:+41-22-382-5489. e-mail: valerie.mclin@hcuge.ch
}

ISSN: Online 1696-3547, Print 0214-6282 


\section{Results}

\section{Cloning of FoxF2}

We isolated a Xenopus laevis $1.1 \mathrm{~kb}$ cDNA (GenBank accession GU 228254) which was homologous to the $X$. tropicalis sequence BC136003.1 (Fig. 1A). The predicted amino acid sequence showed $91 \%$ identity with the $X$. tropicalis sequence (AAI36004.1). The forkhead domain was highly conserved when compared to other vertebrate sequences (Fig. 1B). The protein appears to be highly conserved across species except for additional domains present in the mammalian species (Fig. 1B). FoxF2 amino-acid sequence alignment shows that the conservation between species is greater than the similarities between FoxF1 and FoxF2 in $X$. laevis (Fig. 1C) suggesting a necessary and conserved function for each gene. Consistent with what has been described in other species (Hellqvist et al., 1998, Hellqvist et al., 1996, Pierrou et al., 1994), the most conserved areas of the protein are the DNA (forkhead) binding domain and the C-terminus (Fig. 1B).

\section{Expression of FoxF2 during embryogen- esis of $\mathrm{X}$. laevis}

By in situ hybridization, the earliest detected signal is in the lateral plate mesoderm of the neurula (not shown). By early tailbud stage (Nieuwkoop \& Faber stage 30), faint

Fig. 1. Alignment of FoxF2 with vertebrate homologues. (A) DNA sequence of FoxF2. The translation start site is highlighted in grey. (B) Xenopus laevis FoxF2 predicted amino acid sequence is closely related to other vertebrate sequences. The putative $\mathrm{X}$. laevis and $\mathrm{X}$. tropicalis sequences demonstrate $91 \%$ identity using BLAST (not shown). However, they differ from the mammalian sequences in five domains (underligned) in which the mouse and human sequences are most similar to each other. Notably, the mouse and human sequences contain two domains not present in the amphibian sequences. Additionally, the human sequence contains a domain rich in alanine not present in any of the other species. The following sequences were used for the alignement: mouse: NP_034355, human NP_001443.1, X. tropicalis NP_001093702. (C) Alignment of X.laevis FoxF2 and X.laevis FoxF1 (NP_001084262.1). The two forkhead domains show 3 amino acid differences. The remainder of the proteins show little similarity except for a conserved N-terminus. Alignment of sequences was performed using EBI ClustalW2 (http:// www.ebi.ac.uk/Tools/clustalw2/index.html) and formatting using BOXSHADE 3.21 at the EMBnet website.
A agCTCGGATCCNCTAGTAACGGCCGCCAGTGTGCTGGAATTCGCCCTTGTGTGNATGTAGGGAGACATTC CTTGTACTCCCAGATGAGCACGGAGAAGCACAATCTTTCAGCAGCTCCAATCAGAAGCAGCCCCGCCACA GGGACTGTACAGAGCGCACCGATGAGCCAGCAATCCGCAGCCATGGACACCACCTCCTCTTCTAAGAACA AAAAGCCAAATTCAGGGCTCCGGCGCCCCGAAAAGCCCCCTTATTCCTATATCGCCCTGATAGTCATGGC CATCCAGAGCTCTCCTACCAAAAGACTCACCCTGAGCGAGATCTACCAGTTCCTGCAGGCCCGATTCCCC TTCTTCAGGGGATCCTACCAGGGCTGGAAGAACTCTGTGCGCCACAACCTTTCCCTAAACGAGTGCTTTA TTAAGCTGCCCAAGGGGCTTGGAAGGCCGGGCAAGGGCCACTACTGGACCATTGACCCCGTCAGTGAGTT CATGTTTGAGGAGGGCTCGTTCCGCCGCCGACCCAGGGGCTTTAGAAGAAAATGTCAAGCCCTAAAGTCC ATGTACAGGATGATGAACGGCATTGGCTTCAGCACTTCCATTTTGCCCCAAGGCTTTGATTTCCAGGCCC CACCTGCGTCTCTGGCCTGTCACAGTAATGGCTACAACCTAGACATGATGTCAAACTCTATGGCTGCTGG CTATGATGGCTTAGCCGGGGGGCACCATGTTCCACACATGTCTCCCAACCCTGGCTCTACCTACATGGCC AGCTGTCCTGTGTCTTCCACTGGGGATTACGGGCCAGACAGTAGCAGTAGCCCAGTGCCCTCTTCCCCTG CCATGGCCAGTGCTATGGAATGCCATTCTCCTTACACAAGCCCCACGGCTCACTGGGCATCCTCAGGTGC ATCTTCTTACCTGAAGCAACAGGCCATGCCCCCCAGCAACGCCGCCTCTGCTGCTGGCATCCATTCTGGC GTCTCGCCCTACTCCCTAGAACAGAGTTACCTCCACCAGAACCCCCGGGAGGATCTGTCAGTGGGACTGC CCCGGTACCAGCACCACTCCTCTCCAGTGTGCGACAGGAAAGATTTCGTCCTTAATTTTAACGGGATTTC TTCTTTCCACCCGTCTGCCACTAGTTCCTATTATCACCATCATCACCATCAAAGTGTTTGCCAGGATATA AAGCCCTGCGTGATGAAGGGCGAATTCTGCAGATATCCATCACACNGGCGGCC

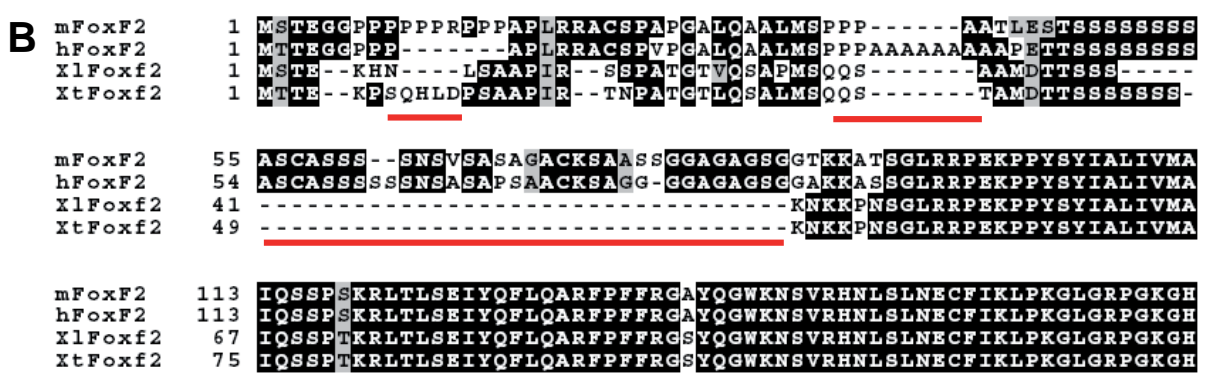

$\operatorname{mFOxF} 2$

hF $\circ$ X 2

$x 1$ F $\circ x \in 2$

173 YWTIDPASEFMFEEGSFRRRPRGFRRKCQALKPUYH

tFoxf 2

YWTIDPASEFMFEEGSERRR PRGFRRKCQALKPMY-RMMNGIGFSTSILPQGFDFQAPP YWTIDPASEFMFEEGSFRRR PRGFRRKCQALKPMY-RMMNGIGFSTSILPQGFDFQAPF-

mFOXF 2

$\mathrm{hF} \circ \mathrm{FF} 2$

$x 1 \mathrm{~F} \circ \mathrm{xf} 2$

$x$ tFoxf 2

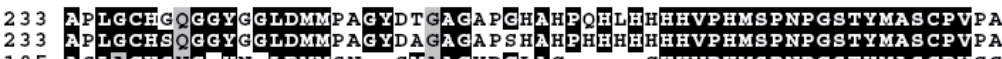

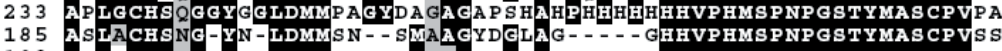
185 ASLACHSNG-YN-LDMMSN - SWAAGYDGLAG----GHHVPHMSPNPGSTYMASCPVS
193 ASITCHSNG-YN-LDMMSN- - SMAGGYDGLAG--- - GHHVPHMSPNPGSTYMASCPVS

$\mathrm{mF} O \mathrm{FF} 2$

hFOXF 2

$x 1$ Foxf 2
x F oxf 2
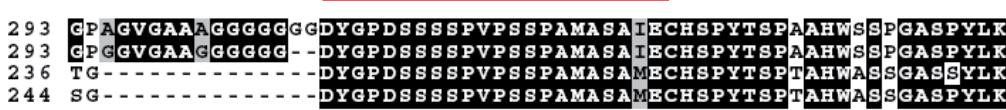

mF $\circ$ F 2

$\begin{array}{ll}\text { hFOXF } 2 & 351 \\ \text { X1FOXf } 2 & 282\end{array}$ PPALTP S SNPAA SAGLHSS MS SY SLEQSYLHQNAREDLSVGLPRYQHHSTPVCDRKDFV

$\begin{array}{ll}x \text { tFoxf } 2 & 290 \\ & \end{array}$

mFOXF 2

$x 1 F \circ x+2$

$x$ tFoxf 2

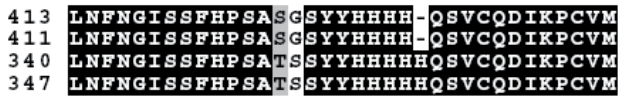

C $\mathrm{XIFOxP1}$

XIPOx 2

1 WTAEIQQP P QPPAQSS PMSAATDKHGGQPSAMESA SCATKTKRTNAGIRRPERPPYSYT
1 WSTEKHNLSAAPIRSSPATGTVQSAPMSOQSAAMDTTS S KNKRPNSGLRRPEXPPYSYI

XIFOXP1

XIFox 2

61 ALIVMAIQSSPTKRLTLSEIYQFLQSRFP FFRGSYQGWKNSVRENLSLNECFIKLPKGLG

$\begin{array}{lll}\text { X1FOXP1 } & 121 \text { RPGKGHYWTIDPASEFMFEEGSFRRRPRGFRRKCQALKPMY SMMNGLGFNH - IPESYSF } \\ \text { XIFOXf2 } & 121 \text { RPGKGHYTIDPASEFMEEGSRRRPRGRRKCQALPMYRMNGIGESTSILEQGDE }\end{array}$

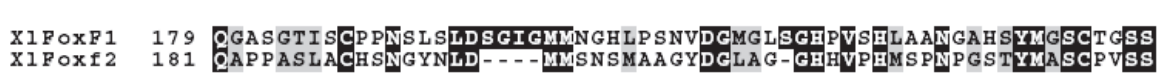

XIFOXF1 239 GGDYSHHDSGSPLLGG - - GGVMEPESVYSSPASAWAPSA S TPYIROQP L SECNTAANPXIFOXf2 236 TGDYGPDSSSPVPSPAKA AMECHSPYTSTAHWASSGAS SYKQQAMPSNAASAAG

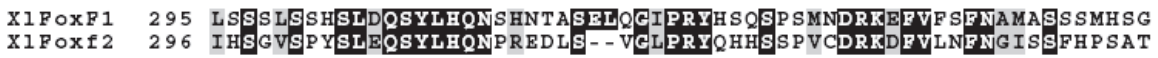

$\begin{array}{lll}\text { XIFOXP1 } & 355 & \text { SGSYYEQQVG-YQDIRPCVM } \\ \text { XIFOXf2 } & 354 & \text { SSYYHEHHHQSVCQDIKPCVM }\end{array}$ 


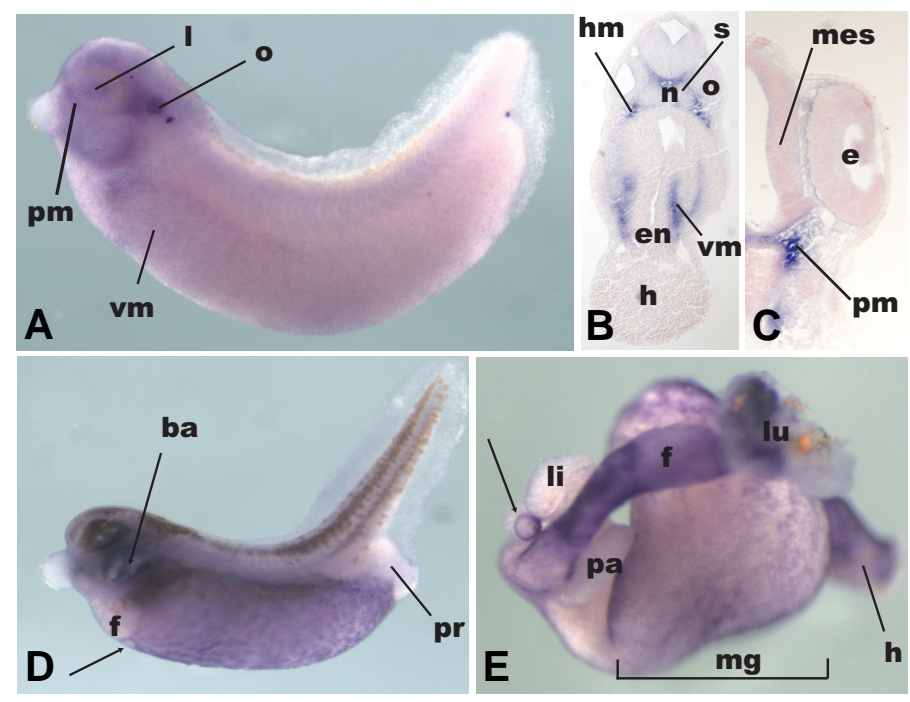

Fig. 2. Embryonic expression of FoxF2. Anterior is to the left and dorsal is to the top. (A) Expression in a tailbud embryo (stage 30). Expression is visible in the otic vesicle (o) and in the periorbital mesenchyme (pm) surrounding the eye, the lens (I) and branchial arches (ba). There is faint expression in the presumptive visceral mesoderm (vm). (B) Section through an embryo a few hours older (stage 35). Expression is noted in the head mesenchyme (hm) surrounding the otic vesicle (o) and the somites (s). Expression is visible in the cranial visceral mesoderm (vm), surrounding the early pharyngeal endoderm (en), (h): heart, (n): notochord. (C) Cross-section through the same embryo, more anterior, highlighting expression in the periorbital mesenchyme (pm), (mes): mesencephalon. (D) In the stage 39 embryo, expression is visible in the branchial arches (ba) and the presumptive visceral mesoderm (vm). The presumptive liver (li) does not express FoxF2. The presumptive gallbladder is highlighted by the circular expression at the anterior ventral expression boundary (arrow). (E) Expression of FoxF2 in the isolated gut of a stage 43 embryo. There is strong expression in the lung (lu) and proximal foregut (f). The gallbladder (arrow) expression is visible, in contrast to the liver (li) and pancreas (pa) which do not express XFoxF2 at this stage. Expression in the midgut ( $\mathrm{mg}$ ) and hindgut ( $h$ ) is characterized by a fine, reticular pattern.

expression in the presumptive visceral mesoderm is noticeable, similar to FoxF1 (El-Hodiri et al., 2001) (Fig. 2A). At this stage, FoxF2 is expressed in the head mesenchyme, in particular in the periorbital region and the mesenchyme surrounding the branchial arches (Fig. 2 A,B), not unlike FoxF1 (El-Hodiri et al., 2001), and in keeping with findings in mouse (Ormestad et al., 2004). Also in keeping with murine expression is the signal detected in the mesoderm beneath the pharyngeal endoderm (Fig. 2B). At this stage, a message is also detected in the otic vesicle (Fig. 2A), which gives rise to the inner ear, and is consistent with expression during early ear development in the mouse (Ormestad et al., 2004). Faint expression is noted in the lens at this stage (Fig. 2A), but a few hours later it is no longer detected (Fig. 2C).

Indeed, at stage 35 , cross-sectional analysis reveals that expression is confined to the head mesenchyme (Fig. 2B) and the contiguous periorbital mesenchyme (Fig. 2C). However, at this stage, there is no expression in the lens or otic vesicle. Conversely, in the mouse, the cochlear precursors do express Foxf2 (Ormestad et al., 2004). We only observed very faint expression in the lining of the mesencephalon and neural tube, unlike the neuroepithelial expression described in rodents (Aitola et al., 2000).

By late tailbud stage (Nieuwkoop and Faber stage 39) (Fig. $2 \mathrm{D})$, there is marked expression in the visceral mesoderm (VM) surrounding the presumptive gut (Fig. 2D) and mimicking the expression of FoxF1. The significance of the reticular pattern noticeable in the VM at this stage is unclear, but might suggest a role in vascular development. Thus, it appears that not all VM cells express FoxF2equally (Fig. 2D). This is an important observation because the VM gives rise to several tissues (blood, muscle, mesenchyme, kidney), and understanding which cell fates require FoxF2 for their specification would aid in unraveling the molecular network governing the formation of mesodermally derived organs. At this stage two areas are notable for their absence of FoxF2 expression: the presumptive liver and the presumptive proctodeum (Fig. 2D). Again, this resembles FoxF1 expression, but differs somewhat from the mouse where Foxf2 is characterized by its distal intestinal expression and associated with colonic malformation and imperforate anus in Foxf2/- animals (Ormestad et al., 2006). At the anterior-most border of visceral mesoderm expression, a distinct circular structure is visualized, which is accepted to be the presumptive gallbladder (Zorn and Mason, 2001).

This finding fits with the distinctive gallbladder expression noticed in the larval stage (Fig. 2E). However, while malformation of the gallbladder has been associated with Foxf1loss-of-function in the mouse (Kalinichenko et al., 2002), this has not been reported for Foxf2. Consistent with the lack of FoxF2 expression in the liver of the late tadpole stage, expression in the liver and pancreas is not noticeable in the larval gut (Fig. 2E). Nevertheless, expression in the presumptive stomach, esophagus and lung is prominent, as it is in the midgut and hindgut, recapitulating the findings in the mouse (Ormestad et al., 2004). We did not appreciate differential expression along the anterior - posterior axis of the larval gut (Fig. 2E), unlike what is described during mouse development (Ormestad et al., 2004). The midgut expression has retained some of the reticular pattern visible in the tadpole (Fig 2 D,E), foreshadowing the adult expression examined below.

\section{Intestinal expression of FoxF2 during metamorphosis}

Metamorphosis in Xenopusspecies is a unique developmental stage under the control of thyroid hormone characterized by distinctive changes in the gastrointestinal tract. First, the intestine undergoes dramatic shortening. Second, the primary epithelium undergoes apoptosis, later giving rise to the secondary epithelium, and these changes probably are in part controlled by the adjacent mesenchyme (Shi and Ishizuya-Oka, 1996). Third, the mesodermally-derived mesenchymal layers undergo rapid expansion from a mono- or bi-layer in the larva to a thick and complex mesenchyme comprised of smooth muscle cells, enteric neurons, vessels, lymphoid cells, subepithelial fibroblasts and mesenchyme. Fourth, the epithelium organizes into folds and troughs similar to the mammalian crypt-villus axis (Shi and IshizuyaOka, 1996). Because of these significant changes, we examined the froglet intestine, immediately following metamorphosis, for FoxF2 expression. At this time, the epithelial folds are starting to form, but the mesenchyme is still very thin. Expression was noted at the mesenchymal epithelial interface in rare cells adjacent to 


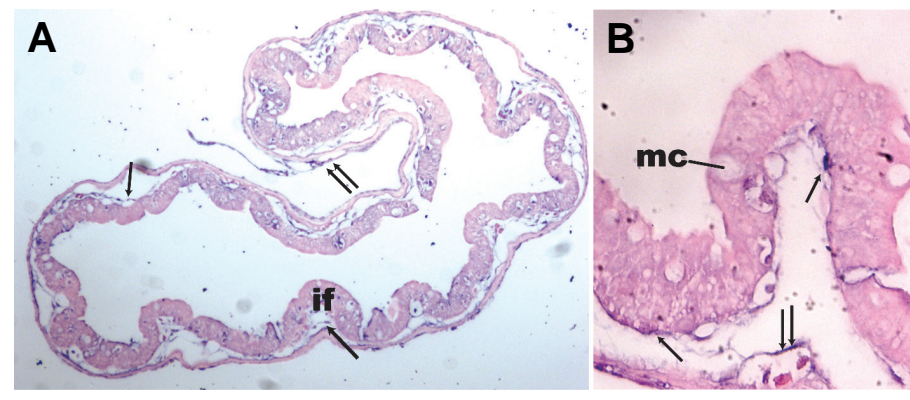

Fig. 3. Expression of XFoxF2 in the froglet intestine. (A) 10x view of a transverse section through the distal intestine of a froglet, immediately post-metamorphosis. The nascent intestinal folds are visible (if). The mesenchymal component of the gut is still underdeveloped. Arrows indicate staining in mesenchymal cells adjacent to the basolateral aspect of the epithelium. Double arrow indicates serosal expression. (B) 40x magnification of a developing intestinal fold. The lumen is to the top. Early mucin expressing cells are visible (mc). Arrows indicate expression at the epithelial-mesenchymal interface. Double arrow indicates expression in early blood vessel (blood cells in lumen).

the epithelium, both in the developing folds and troughs (Fig. 3 $A, B)$. Further, expression was noted in the wall of the enteric blood vessels (Fig. 3B). In light of the adult expression detailed below, these findings are significant, because they hint at the early precursors of the adult mesenchyme.

\section{Expression of FoxF2 in the adult intestine of $\mathrm{X}$. laevis}

In the adult intestine, FoxF2 expression is confined to the mesenchymal and serosal layers, which contrasts with descriptions in rodents in which serosal expression was not observed except in the developing lung (Aitola etal., 2000). In mouse, Foxf2 is expressed in at low levels in the subepithelial mesenchyme and muscularis externa (Aitola et al., 2000). In contrast, there is no expression in the thick muscularis of the adult frog. Rather, the expression is confined to the mesenchymal layer with a clear interface between mesenchyme and muscularis (Fig. 4 A-D). This diffuse pattern of mesenchymal expression is similar to what has been reported for murine Foxf2, and contrasts with murine Foxf1 which expression is strongest at the epithelial-mesenchymal interface (Aitola et al., 2000). Of note, we examined expression of FoxF1 in adult intestine and found a very similar expression pattern to FoxF2 with little differential expression along the radial axis (not shown). Both the mesenchyme at the base of the troughs and in the intestinal folds show expression. The vessel walls in the mesenchyme also express FoxF2, something which has been reported in mouse but not shown in Xenopus (Ormestad et al., 2004). In the distal intestine, there is marked expression in both the mesenchyme and vessel walls. Distal intestinal expression is also very similar to FoXF1 (not shown). We did not detect a FoxF2 message in the adult lung or liver by RT-PCR or in situ hybridization. This is in slight contrast to what has been reported in mouse, where there is a low level of expression by in situ hybridization in the adult lung (Aitola et al., 2000). Consistent with previous reports in the mouse (Aitola et al., 2000, Ormestad et al., 2004), however, is the fact that both on sections and whole-mounts in adults and embryos, a long exposure time was required, suggesting that FoxF2 is expressed at low levels.

\section{Discussion}

We have shown that FoxF2 is expressed in the mesenchyme of the developing and adult gastrointestinal tract of Xenopus laevis. Unlike what has been previously reported in mouse, we see similar expression in both the anterior and posterior aspects of the embryonic and adult gastrointestinal tract. It is possible that the differential requirement between anterior and posterior is a mammalian adaptation, and that the uniquely mammalian do-
Fig. 4. Expression of XFoxF2 in the adult frog intestine. (A) Proximal intestine (4x magnification) showing FoxF2 expression in the subepithelial mesenchyme, between the epithelium (e) and the muscularis propria $(\mathrm{m})$. There is light staining in the serosa (s). (B) 10x magnification of (A) highlighting expression in the mesenchyme of the intestinal fold (if) and surrounding mesenchymal vessels (arrow). The sections in $(A, B)$ were counterstained using eosin. (C) $4 x$ magnification of the distal intestine. The distal gut mesenchyme is less compact than proximally, butFoxF2 expression is also confined to the mesenchyme. The dark staining in the intestinal folds is pigment (p). Faint serosal expression is also noted (s). (D) 10X magnification of (C) highlighting expression in the mesenchymal stalk of the intestinal folds. The distal intestine is characterized by abundant mucin-producing cells (mc). The empty lumina are either vascular or lymphatic (I). Nuclear Fast Red was used for counterstaining of the distal intestine, highlighting the mesenchymal nuclei $(C$, $D)$. Pigment is visible in the intestinal folds and trough; these cells are accepted to be melanophores derived from the neural crest (Nieuwkoop, 1994).
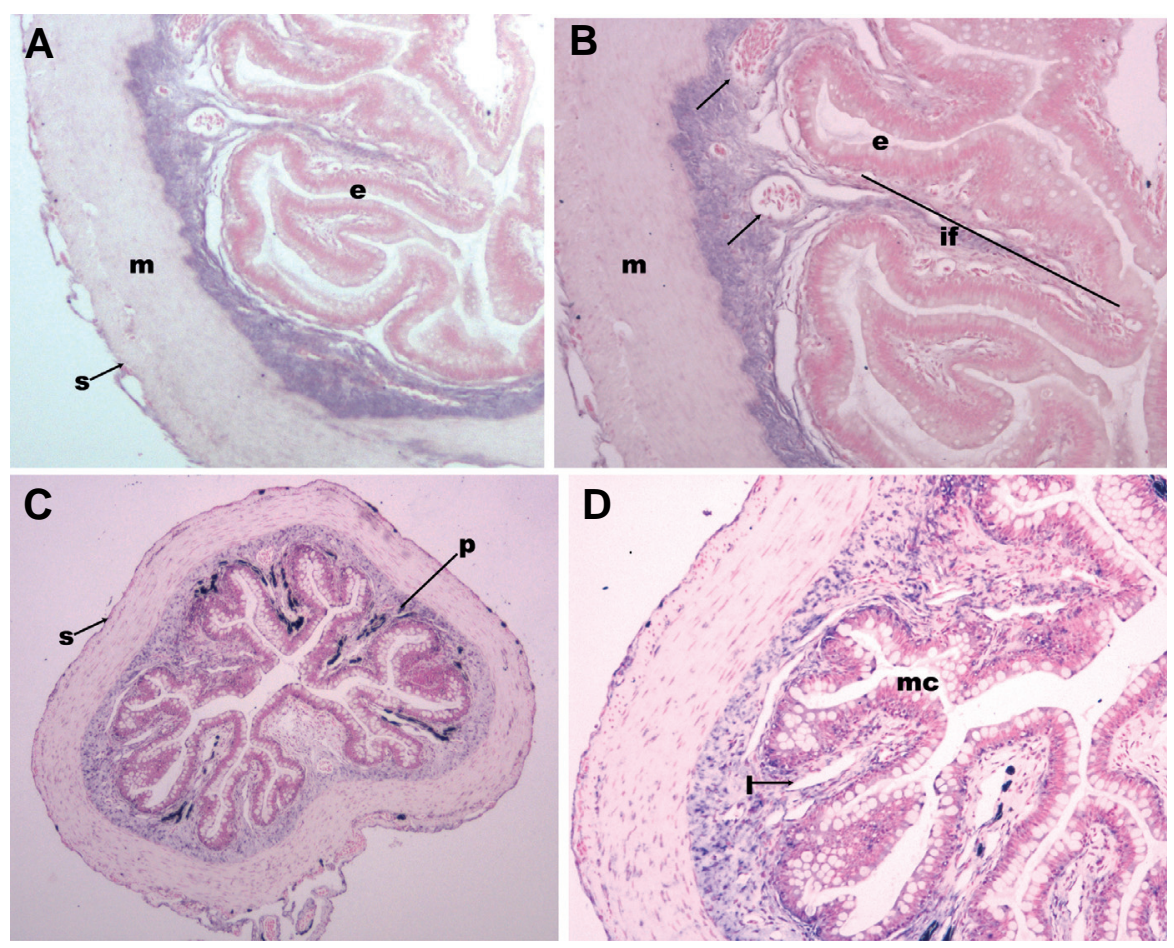
mains identified in the protein sequence participate in the regulation of this differential expression, something which remains to be tested. The highly conserved sequence between Xenopus and mammals suggests that the function of FoxF2has been selected for in development. However, the amino-acid sequences of FoxF1 and FoxF2 are very dissimilar outside the forkhead domain, suggesting that rather than being redundant, these proteins have distinct and necessary functions.

The other significant finding from these studies is the vascular expression observed in the adult, and the reticular pattern noted in the embryo, pointing to a role for FoxF2 in vascular development. Although it has been shown in mouse that Foxf1 is required for vessel formation (Astorga and Carlsson, 2007), the role of FoxF2 in this process has not been elucidated.

Likewise, gallbladder expression of FoxF2 is a novel finding, not previously reported in mouse, but described for Foxf1. This is intriguing because the liver and pancreas, which also derive from the foregut, do not express FoxF2. Since one of the striking characteristics distinguishing the gallbladder from its adjacent structures is its tubular shape, this expression pattern raises the question of the contribution of FoxF genes to lumen formation. Indeed, in Foxf2/- animals, lumen formation is severely impeded by excessive epithelial proliferation (Ormestad et al., 2006).

From a molecular perspective, the expression of FoxF2 in the mesenchyme of Xenopus laevis appears very similar to BMP-4 and BMP-1 expression (Ishizuya-Oka and Shi, 2007). During embryonic development, $B M P-4$ is known to be upstream of FoxF1 both in vascular and visceral mesoderm development (Astorga and Carlsson, 2007, Ormestad etal., 2006, Tseng et al., 2004). The coincident expression of the two genes in the visceral mesenchyme suggests that this regulatory paradigm may be conserved in the adult.

Further, because Xenopusmetamorphosis is exquisitely regulated by thyroid hormone (TH), the finding that FoxF2-expressing tissue expands vastly following metamorphosis raises the possibility that FoxFgenes may be in part regulated by $\mathrm{TH}$, something which has not been investigated to date. Xenopus laevis is an attractive model to test this hypothesis for two reasons. First, metamorphosis can be induced experimentally by adding $\mathrm{TH}$ to the frog water (Shi and Brown, 1993). Second, since very few mesenchymal cells express FoxF2 immediately following metamorphosis, the study of post-metamorphosis mesenchymal proliferation and differentiation may yield insight into putative, intestinal, mesenchymal stem cell regulation. Moreover, this hypothesis may be relevant to mammals since the changes observed at metamorphosis in amphibians have been compared to mammalian birth, which is also associated with a surge in thyroid hormone levels (Crockford, 2003, Tata, 1993). Indeed, mesenchymal proliferation in Xenopus laevis is under the control of TH (Shi and Ishizuya-Oka, 1996), and mice lacking the thyroid receptor $\alpha$ or $\beta$ show abnormal development of the mesenchymal component of the intestine with concomitant aberrant epithelial proliferation and differentiation (Plateroti et al., 1999).

In summary, we illustrate that FoxF2 expression shows similarities and differences compared to murine Foxf2. During development and adulthood, it is expressed in both the proximal and distal intestine, unlike what has been reported in mouse. Second, its expression in the vasculature and gallbladder are other novel findings. Importantly, it is expressed in a thin layer of intestinal mesenchymal cells at metamorphosis, presumably the precursors of the abundant FoxF2-expressing adult mesenchymal fibroblasts. Future studies are needed to determine the relationship between $\mathrm{TH}$ and FoxF2and whether it can serve as a mesenchymal stem cell marker.

\section{Materials and Methods}

\section{PCR}

FoxF2 was PCR amplified using degenerate primers for forkhead box (F: IVMAIQ, R: EFMFEEG) on cDNA obtained from adult Xenopus laevis intestinal mesenchyme. The resulting bands were TOPO-TA cloned and sequenced. Using specific primers (F:VYVGRH, R:DIKCPVM) for the $X$ tropicalis sequence (BC136003), we isolated a 1100 base pair sequence including the ATG (Fig. 1) from Xenopus laeviscDNA. A shorter sequence (approximately 500bp) was inserted into pBluescript to make the in situ probe.

\section{Isolation of adult Xenopus organs, froglet intestine and embryos}

Adult animals and froglets were anesthetized in $0.05 \%$ benzocaine for 30 minutes according to conventional methods. After a midline incision, the intestine was isolated from the gastroeosophageal junction to the rectum. The intestine was flushed using cold PBS and then fixed in 10\% formalin overnight. Lung and liver was removed by clipping the vessels at the hilum. Embryos were collected as previously described (Sive, 2000).

\section{In situ hybridization on whole mount and sections}

In situ on whole embryos and isolated guts were performed as previously published (McLin et al., 2008). In situhybridization on sections of froglet and adult gut were performed in the following manner. First, paraffin was removed using absolute alcohol and then rinsed well and placed in RNAse free water. Next, enzymatic digestion with Ficin 1:50 was performed at room temperature for 15 minutes. Endogenous peroxidase was blocked 15 minutes at room temperature and rinsed well with distilled water followed by a rinse in RNAse free water. Sections were then dehydrated through graded alcohols and slides allowed to dry completely. $30 \mu \mathrm{L}$ of probe diluted in hybridization solution (same as for whole mount) was applied to each slide. Incubation was performed in a humid chamber at 37 degrees Celsius overnight. On day 2, slides were rinsed in 4XSSC buffer followed by 2XSSC buffer and distilled water. No blocking step was used. After patting them try, the slides were incubated at room temperature for one hour with the anti-DIG antibody (Roche). Following additional washes, NBT/BCIP was used as a chromagen. It was allowed to develop for several hours, checking microscopically at regular intervals to determine desired end point. Sections were counterstained with either eosin or nuclear Fast Red.

\section{Acknowledgements}

VAM is supported by the NIH (K08DK078656) and a Young Investigator Award from the CDHNF. MJ is supported by the Retinal Research Foundation. This work was supported in part by Public Health Service Grant DK56338, which funds the Texas Medical Center Digestive Diseases Center (DDC). The authors thank Dorene Rudman of the DDC Morphology Core for her expert technical assistance, and Travis Bailey and Rainer Lanz for helpful discussions.

\section{References}

AITOLA, M., CARLSSON, P., MAHLAPUU, M., ENERBACK, S. and PELTO HUIKKO, M. (2000). Forkhead transcription factor foxf2 is expressed in mesodermal tissues involved in epithelio-mesenchymal interactions. Dev Dyn 218 136-149

ASTORGA, J. and CARLSSON, P. (2007). Hedgehog induction of murine vasculogenesis is mediated by foxf1 and bmp4. Development 134: 3753-3761. 
CLEVIDENCE, D.E., OVERDIER, D.G., TAO, W., QIAN, X., PANI, L., LAI, E. and COSTA, R.H. (1993). Identification of nine tissue-specific transcription factors of the hepatocyte nuclear factor $3 /$ forkhead dna-binding-domain family. Proc Nat/ Acad Sci USA 90: 3948-3952.

CROCKFORD, S.J. (2003). Thyroid rhythm phenotypes and hominid evolution: A new paradigm implicates pulsatile hormone secretion in speciation and adaptation changes. Comp Biochem Physiol A Mol Integr Physiol135: 105-129.

EL-HODIRI, H., BHATIA-DEY, N., KENYON, K., AULT, K., DIRKSEN, M. and JAMRICH, M. (2001). Fox (forkhead) genes are involved in the dorso-ventral patterning of the Xenopus mesoderm. Int J Dev Bio/45: 265-271.

HELLQVIST, M., MAHLAPUU, M., BLIXT, A., ENERBACK, S. and CARLSSON, P. (1998). The human forkhead protein freac-2 contains two functionally redundant activation domains and interacts with tbp and tfiib. JBio/Chem273: 2333523343.

HELLQVIST, M., MAHLAPUU, M., SAMUELSSON, L., ENERBACK, S. and CARLSSON, P. (1996). Differential activation of lung-specific genes by two forkhead proteins, freac-1 and freac-2. J Biol Chem 271: 4482-4490.

ISHIZUYA-OKA, A. and SHI, Y.B. (2007). Regulation of adult intestinal epithelial stem cell development by thyroid hormone during Xenopus laevismetamorphosis. Dev Dyn 236: 3358-3368.

KALINICHENKO, V.V., ZHOU, Y., BHATTACHARYYA, D., KIM, W., SHIN, B., BAMBAL, K. and COSTA, R.H. (2002). Haploinsufficiency of the mouse forkhead box $\mathrm{f1}$ gene causes defects in gall bladder development. JBio/Chem277: 12369-12374.

MAHLAPUU, M., ENERBACK, S. and CARLSSON, P. (2001). Haploinsufficiency of the forkhead gene foxf1, a target for sonic hedgehog signaling, causes lung and foregut malformations. Development 128: 2397-2406.

MAHLAPUU, M., ORMESTAD, M., ENERBACK, S. and CARLSSON, P. (2001). The forkhead transcription factor foxf1 is required for differentiation of extraembryonic and lateral plate mesoderm. Development 128: 155-166.

MAHLAPUU, M., PELTO-HUIKKO, M., AITOLA, M., ENERBACK, S. and CARLSSON, P. (1998). Freac-1 contains a cell-type-specific transcriptional activation domain and is expressed in epithelial-mesenchymal interfaces. Dev Bio/202: 183-195.

MCLIN, V.A., HU, C.H., SHAH, R. and JAMRICH, M. (2008). Expression of complement components coincides with early patterning and organogenesis in
Xenopus laevis. Int J Dev Bio/52: 1123-1133.

NIEUWKOOP, D., FABER, J. (1994). Normal table of Xenopus laevis (daudin). Garland Publishing Inc, New York.

ORMESTAD, M., ASTORGA, J. and CARLSSON, P. (2004). Differences in the embryonic expression patterns of mouse foxf 1 and -2 match their distinct mutant phenotypes. Dev Dyn 229: 328-333.

ORMESTAD, M., ASTORGA, J., LANDGREN, H., WANG, T., JOHANSSON, B.R. MIURA, N. and CARLSSON, P. (2006). Foxf1 and foxf2 control murine gut development by limiting mesenchymal wnt signaling and promoting extracellular matrix production. Development 133: 833-843.

PIERROU, S., HELLQVIST, M., SAMUELSSON, L., ENERBACK, S. and CARLSSON, P. (1994). Cloning and characterization of seven human forkhead proteins: Binding site specificity and dna bending. EMBO J13: 5002-5012.

PLATEROTI, M., CHASSANDE, O., FRAICHARD, A., GAUTHIER, K., FREUND, J.N., SAMARUT, J. and KEDINGER, M. (1999). Involvement of t3ralpha- and beta-receptor subtypes in mediation of $\mathrm{t} 3$ functions during postnatal murine intestinal development. Gastroenterology 116: 1367-1378.

SHI, Y.B. and BROWN, D.D. (1993). The earliest changes in gene expression in tadpole intestine induced by thyroid hormone. J Biol Chem 268: 20312-20317.

SHI, Y.B. and ISHIZUYA-OKA, A. (1996). Biphasic intestinal development in amphibians: Embryogenesis and remodeling during metamorphosis. Curr Top Dev Bio/32: 205-235.

SIVE, H.L.G., R.M; HARLAND, R. M. (2000). Early development of Xenopus laevis. A laboratory manual. Cold Spring Harbor Laboratory Press, Cold Spring Harbor, New York.

TATA, J.R. (1993). Gene expression during metamorphosis: An ideal model for post-embryonic development. Bioessays 15: 239-248.

TSENG, H.T., SHAH, R. and JAMRICH, M. (2004). Function and regulation of foxf1 during Xenopus gut development. Development 131: 3637-3647.

ZAFFRAN, S., KUCHLER, A., LEE, H.H. and FRASCH, M. (2001). Biniou (foxf), a central component in a regulatory network controlling visceral mesoderm development and midgut morphogenesis in Drosophila. Genes Dev 15: 2900 2915.

ZORN, A.M. and MASON, J. (2001). Gene expression in the embryonic Xenopus liver. Mech Dev 103: 153-157.

\section{Further Related Reading, published previously in the Int. J. Dev. Biol.}

See Special Issue Pattern Formation edited by Michael K. Richardson and Cheng-Ming Chuong at:

http://www.ijdb.ehu.es/web/contents.php?vol=53\&issue=5-6

Expression of FoxP2 during zebrafish development and in the adult brain Rina Shah, Olga Medina-Martinez, Li-Fang Chu, Rodney C. Samaco and Milan Jamrich Int. J. Dev. Biol. (2006) 50: 435-438

Fox (forkhead) genes are involved in the dorso-ventral patterning of the Xenopus mesoderm

H El-Hodiri, N Bhatia-Dey, K Kenyon, K Ault, M Dirksen and M Jamrich

Int. J. Dev. Biol. (2001) 45: 265-271

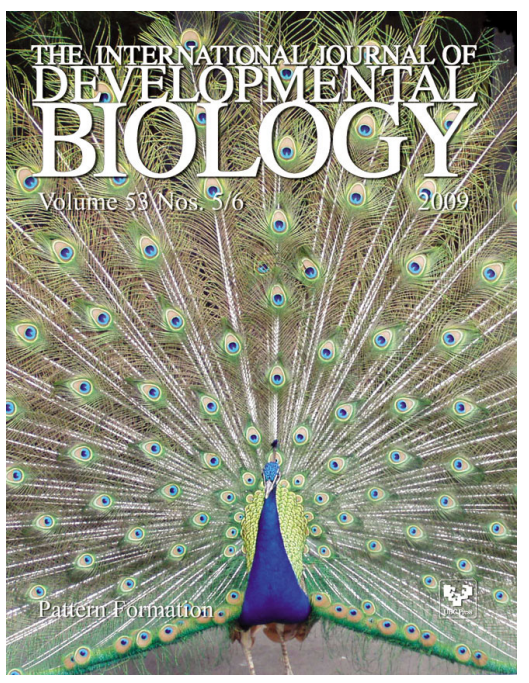

